\title{
Valoración económica del daño ocasionado por el incendio Área de Conservación Privada "Milpuj - La Heredad", Magdalena, Amazonas.
}

\section{Economic evaluation of the damage caused by the fire Private Conservation Area "Milpuj - La Heredad", Magdalena, Amazonas.}

\author{
Carlos Alberto Casiano Inga ${ }^{1}$, Wagner Guzmán Castillo ${ }^{2}$, Jonathan Alberto Campos Trigoso ${ }^{3}$
}

\section{RESUMEN}

Los ecosistemas de bosque seco forman parte de los valles interandinos del noreste de Perú, y son considerados como una de las fronteras biogeográficas más importante de los Andes, que alberga especies endémicas de flora y fauna. Sin embargo, debido a la presión antrópica, como la quema para ampliar la frontera agrícola y ganadera, tala, cacería, invasiones, etc.; estos ecosistemas están siendo reducidos y amenazados, y por ende, los servicios ecosistémicos que brindan. Por lo cual, a pesar de los esfuerzos de titulares privados por invertir en la conservación de la diversidad biológica a través de modelos de aprovechamiento sostenible y la restauración de los mismos, aún se evidencia la desvinculación del cuidado de la biodiversidad de terceras personas, que constantemente atentan contra estos espacios. En consecuencia, se propone la valoración económica de los daños a los recursos naturales para cuantificar los daños y perjuicios causados sobre los valores de uso y no uso, en términos monetarios, para la compensación adecuada, por las pérdidas en recursos productivos y/o por gastos de limpieza y restauración del área, debido al incendio ocasionado en el ACP Milpuj - La Heredad. El análisis del valor económico del daño se hizo a través de la suma de los costos del daño, costos de las medidas de restauración y del valor económico de los servicios ecosistémicos del bosque seco; obteniendo la cantidad total de S/.210, 279. 67, y S/. 8,963.33/ha.

Palabras clave: Valoración económica del daño, incendio, Área de Conservación Privada (ACP), costos del daño, costos de restauración.

\begin{abstract}
Dry forest ecosystems are part of the inter-Andean valleys of northeastern of Peru, and they are considered one of the most important biogeographical borders of the Andes, that hosts endemic flora and fauna species. Nevertheless, due to anthropic pressure, such as burning to expand agricultural and livestock frontier, logging, hunting, invasions, etc.; these ecosystems are being reduced and threatened, and therefore, ecosystem services that provide. Despite the efforts of private owners, who invest in the conservation of biodiversity through sustainable models, there is still evidence of the disconnection between biodiversity and human being, who constantly cause pressure on these spaces. Consequently, the economic valuation of the damages on natural resources is proposed to quantify the damages and losses caused on use and non-use values, in monetary terms, to the adequate compensation, due to losses in productive resources and/or expenses for cleaning and restoring the area that was set on fire in Milpuj - La Heredad PCA. The analysis of the economic value of damage was obtained through the sum of damage costs, restoration costs and economic valuation of environmental services of the dry forest; obtaining S/.210,279.67 as the total quantity, and S/. 8,963.33/ha.
\end{abstract}

Keywords: Economic valuation of damage, dry forest fire, Private Conservation Area (PCA), damage cost, restoration cost.

${ }^{1}$ Especialista Ambiental. Dirección Ejecutiva de Gestión Ambiental de la Autoridad Regional Ambiental de Amazonas. Correo electrónico: carlosbeto11@hotmail.com

${ }^{2} \mathrm{Mg}$., Coordinador de la OD SUNASS Amazonas. Correo electrónico: wagner.castillo@untrm.edu.pe

${ }^{3} \mathrm{Mg}$., Director Ejecutivo del Instituto de Investigación en Negocios Agropecuarios - INNA. Correo electrónico: jonathan.campos@untrm.edu.pe 


\section{INTRODUCCIÓN}

La figura legal de Área de Conservación Privada (ACP) se incluyó en la Ley de Áreas Naturales Protegidas (1997) dando inicio a la conservación voluntaria en el Perú (MINAM, 2014). Estas ACP son reconocidas como tales por el Estado, a través de una Resolución Ministerial del MINAM, por sus características ambientales, biológicas, paisajísticas u otras análogas; así como por su contribución a la conservación de la diversidad biológica, incremento de la oferta para la investigación científica, la educación y al turismo especializado (SPDA, 2012).

En los últimos años, la conservación privada ha cobrado mayor importancia, cada vez son más personas, comunidades, organizaciones no gubernamentales y empresas que apuestan por la conservación de la diversidad biológica; es así que actualmente se cuenta con 122 Áreas de Protección Privada (ACP) en el país con un área total 366113.50 ha, de las cuales 15 (12.3\%) están ubicadas en el departamento de Amazonas, cubriendo un total de 12 6732.7 ha $(34.62 \%)$ del territorio nacional (SERNANP, 2018; NCI, 2017).

El ACP Milpuj - La Heredad, ubicada en la margen derecha del valle medio del Utcubamba, entre los 1830 - 1950 m.s.n.m., en el sector el Chillo, distrito del Magdalena, provincia de Chachapoyas, departamento de Amazonas (Epiquién, 2009); es una de estas ACP, que fue reconocida como tal por un periodo de diez (10) años, con Resolución Ministerial No 164-2011-MINAM, del 26 de julio del 2011; con el objetivo de conservar parte de los bosques y matorrales secos de la cuenca media del río Utcubamba, a través de estrategias de conservación privada y el manejo sostenible de sus recursos (SERNANP, 2018).

El ecosistema que alberga, bosques y matorrales secos, está siendo aceleradamente reducido debido a la quema estacional de los bosques y matorrales para ampliar la frontera agrícola y ganadera, tala selectiva para uso doméstico de leña y construcción, cacería de mamíferos grandes como venados, armadillos y otros (Epiquién, 2009; GOREA, ARA \& NCI, 2013), y que sumado a los efectos del cambio climático, en especial las sequías, están trayendo consigo la degradación de sus servicios ecosistémicos (GOREA,ARA \& NCI, 2013).

Estos problemas no fueron ajenos, cuando a mediados del año 2000, los hoy propietarios del ACP Milpuj - La Heredad se mudaron a vivir, encontrando sus áreas deforestadas e invadidas por cazadores furtivos. Aunque la incidencia se ha reducido considerablemente, en la actualidad aún se registran invasiones a su propiedad privada, encontrando árboles que son talados para postes de cercos o ser usados como leña; también se ha visto las pretensiones de atentar contra el ACP instalando alambrado de manera ilegal.

Aunque, como medida cautelar, han venido manifestando su preocupación a las instancias con competencias ambientales de la región Amazonas, por atentados contra la integridad del ACP, no han recibido el soporte institucional y legal para hacer frente a estos problemas; a pesar de que el Ministerio del Ambiente (MINAM), cuando la reconoció como $\mathrm{ACP}$, revaloró los esfuerzos de sus propietarios por invertir en conservación, teniendo el respaldo y seguridad jurídica del Estado (SPDA, 2012).

El 21 de noviembre de 2017 a las 10:00 a.m. se desató un grave atentado contra la diversidad biológica, un incendio intencionado en la zona SUR, con propagación de la parte baja hacia la alta, que se extendió hasta 5:30 p.m. en la que el siniestro fue controlado. Se tuvo una aproximación de los daños: 30 ha afectadas, de las cuales 4 ha incluye el ACP Milpuj - La Heredad; afectación de plantas forestales, especies endémicas, plantas en peligro de extinción, 04 colmenas de abejas del apiario, reservorio cubierto con geomembrana y aproximadamente 5 mil especies forestales nativas sembradas en los últimos cinco años; tal cual se manifestó en la parte policial 166-2017 el 22 de noviembre de 2017.

Si bien, esa fue una primera mirada exhaustiva de los daños ocasionados por el incendio intencionado; a través de la valoración económica se busca estimar, en términos monetarios, la pérdida del bienestar aproximado de los daños.

Con el objetivo de conocer los daños reales por el incendio provocado en el ACP Milpuj - La Heredad, se realizó la valoración económica, la cual tiene como fin último el análisis Costo - Beneficio para la cuantificación de la indemnización necesaria por el daño ambiental percibido (PUCP, 2013) o el programa de restauración preferido después de un daño ambiental.

\section{MATERIAL Y MÉTODO}

En general, podemos decir que el valor económico de los bienes ambientales tiene dos componentes, el valor de uso y el valor de no uso (Labandeira et al., 2007). Para este caso en particular, el valor de uso se deriva del uso que hacen los propietarios del ACP 
Milpuj - La Heredad de los recursos que le brinda este ecosistema de bosque seco. Los valores de no uso se derivan de las motivaciones de existencia, herencia y altruismo que pueden tener los propietarios y las personas que visitan esta ACP.

La metodología para la aproximación del valor económico del daño por el incendio intencionado del Área de Conservación Privada Milpuj - La Heredad en el año 2017, está bajo la premisa de que el valor del daño es equivalente a los componentes de valor de uso y valor de no uso (ver Gráfico 01).

\section{Valor económico total de los bienes ambientales $=$} valor de uso + valor de no uso

Para determinar el valor de uso se utilizaron dos métodos: 1) método de costos de daño, sobre las principales actividades económicas calificadas como significativas (apicultura, producción de tara, reservorio), y 2) método de costos de restauración, gastos incurridos para mitigar los efectos del incendio intencionado (reforestación con plantones de cedro kuelapense, tara, aliso, balsa, mutuy, guayo, pavicuna, huarango y eucalipto).

Para obtener el valor de no uso, se utilizó los resultados de la investigación de Costanza et al. (1997), "valor de capital natural y servicios ecosistémicos del mundo". Por lo tanto, la ecuación para obtención del valor económico del daño sería equivalente a:

Valor económico del daño $\cong$ costos de daños + costos de restauración + valor de no uso del ecosistema de bosque seco

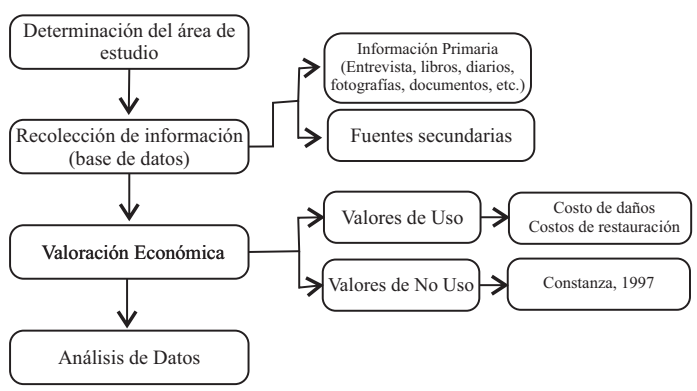

Gráfico 01. Metodología para la aproximación del valor económico del daño por el incendio intencionado del ACP.

Fuente: Elaboración propia.

Haciendo uso de imágenes satelitales, GPS y con el apoyo de un dron, se determinó con exactitud el área afectada por el incendio provocado, llegando a obtener una afectación de 23.46 ha (ver Gráfico 02). Sobre la base de esta área se realiza la valoración económica del daño.

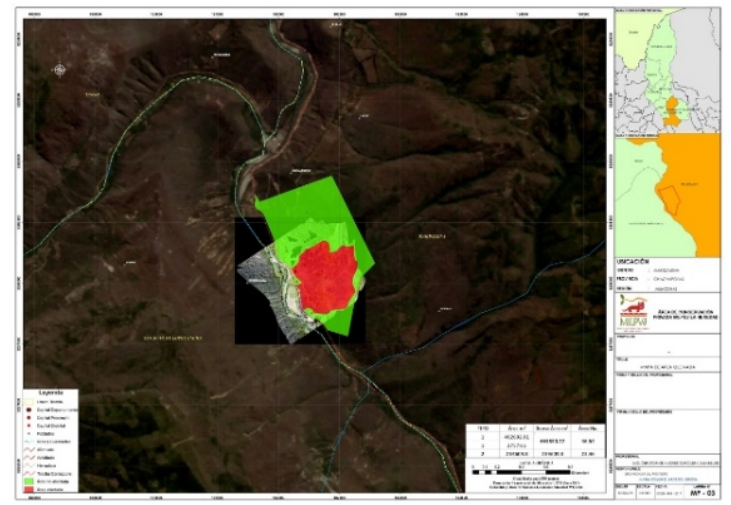

Gráfico 02. Mapa de área afectada por el incendio en el ACP Milpuj - La Heredad. Fuente: Corcuera, 2018.

\section{Métodos de los costos de daño}

Este método es usado para estimar el valor económico de bienes y servicios derivados de recursos naturales que son usados como insumos en la producción de bienes finales (MINAMBIENTE, 2003).

El análisis de este método se basa en dos tipos de beneficios o costos. Primero, si cambia la calidad o el precio a los consumidores del bien final, habrá cambios del excedente del consumidor. Segundo, si cambia la productividad o los costos de producción, habrá cambios del excedente del productor (MINAMBIENTE, 2003).

\section{Modelo}

$\mathrm{V}$ : Factores fijos de producción

$\mathrm{K}$ : Factores variables de producción q: Calidad ambiental

Ante un deterioro del bien "q" que inciden en la producción, los costos de producción serán mayores, entonces una subida en el costo del factor ambiental se reflejará en una disminución del bienestar del productor, lo que será tomado como la valoración de la pérdida de la calidad ambiental.

\section{Aplicación de la metodología}

Se recolectaron datos teniendo en cuanta cómo los cambios en la cantidad y la calidad del recurso natural afectan los siguientes ítems:

- Los costos de producción de apicultura y de tara.

- Oferta y demanda de la producción de tara y miel de abeja.

- Oferta y demanda del turismo, el cual no fue considerado en la valoración económica por ser una variable poco significativa hasta la fecha. 
De la aplicación de la metodología, donde el costo del daño llega a ser igual a la suma de los costos por el cambio en la productividad de tara y apicultura, se obtuvo el costo del daño sobre los mismos (para mayor detalle de los costos ver Anexos):

Tabla 1: Costo del daño sobre la producción de apicultura y tara

\begin{tabular}{|c|c|}
\hline $\begin{array}{c}\text { Actividad económica } \\
\text { / bienes dañados }\end{array}$ & costos del daño \\
\hline Apicultura & S/ 25,370.00 \\
\hline Producción de tara & S/ 20,557.41 \\
\hline Costo del Daño & S/. 45,927.41 \\
\hline
\end{tabular}

\section{Método del costo de restauración}

La restauración de un ecosistema realizado para compensar la degradación puede ser vista como una técnica de reemplazo. El ecosistema proporciona un entorno sostenible para las especies que viven allí y es por tanto un recurso valioso. El valor puede ser establecido con el uso de un estudio de costo de reposición, donde se estima el costo de la restauración del ecosistema.

Considerar el gasto por restaurar y devolverle al ecosistema su estado original causado por la intervención antrópica; es una aproximación del valor de los beneficios ambientales alteados (Pérez, 2008). Para lo cual se identificaron los recursos dañados, y se estimó los gastos por reposición y/o restauración, a través de actividades de reforestación.

Tabla 2: Costos de restauración por deforestación

\begin{tabular}{|c|c|}
\hline Actividad de restauración & Costos de restauración total \\
\hline Reforestación & $\mathrm{S} / 13,682.75$ \\
\hline Costo de Restauración & $\mathrm{S} / \mathbf{1 3}, \mathbf{6 8 2 . 7 5}$ \\
\hline
\end{tabular}

\section{Obtención de valor de no uso}

De acuerdo al mapa global del valor de los servicios ecosistémicos de Costanza et al. (1997), tales como regulación del clima, regulación hídrica, suministro de agua, control de la erosión, reciclaje de nutrientes, polinización, hábitat y refugio, producción de alimentos, recursos genéticos, recreación, cultura, entre otros; el valor económico anual promedio para este tipo de ecosistema, bosque tropical estacionalmente seco es de $\$ 2,007 /$ ha, que en moneda nacional equivale alrededor de $\mathrm{S} / 6,422.40 /$ ha, y que multiplicado por el área de afectación (23.46 ha), el valor económico del valor de no uso equivale a $\mathbf{S} /$. $150,669.504$

Valor económico de daño a causa del incendio provocado en el ACP Milpuj - La Heredad

Valor Económico del daño $=$ Costos de los daños + Costos de restauración + valor de los servicios ecosistémicos.

Valor económico del daño $=$ S/. 45,927.41 + S $/$. $13,682.75+150,669.504$.

Valor económico del daño $=\mathrm{S} / . \mathbf{2 1 0}, 279.67$

\section{Características de la zona de estudio}

Milpuj se encuentra ubicado en el valle medio de la cuenca del río Utcubamba, dentro del extenso corredor interandino de los últimos bosques y matorrales semisecos del país (Epiquién, 2009). Reconocida como ACP Milpuj - La Heredad, interpola la conservación con el turismo y el desarrollo de actividades productivas sostenibles.

Se caracteriza por poseer dos tipos de ecosistemas: Bosque Seco Premontano Tropical (bs-PT) y el Bosque Seco Montano Bajo Tropical (bs-MBT), las cuales determinan la geomorfología que albergan una biodiversidad única de un ecosistema amenazado constantemente; con más de 80 aves registradas, de las cuales 05 han sido identificadas como endémicas, 16 tipos de mamíferos, 02 de los cuales son felinos en estado de vulnerabilidad. Los valores de la biodiversidad que otorga esta área son los valores ecológicos, florísticos, faunísticos, ambientales, culturales, turísticos, entre muchos otros.

Está determinada por dos temporadas bien definidas, la temporada seca de julio a setiembre, y la temporada lluviosa de octubre a mayo, predominando un clima ligeramente húmedo y templado cálido.

El ACP Milpuj - La Heredad no sólo alberga una rica biodiversidad, sino también cuenta con un legado histórico, el Qhapaq Ñam o camino inca que recorre parte de la propiedad en sentido Norte-Sur, con una extensión reportada de $920 \mathrm{~m}$ de largo aproximadamente, y un ancho variable de 2 a $4 \mathrm{~m}$.

Los ecosistemas secos y semisecos interandinos son considerados como áreas prioritarias para la conservación, debido a sus características de 
endemismo y rareza; además por las constantes amenazas antrópicas que sufren.

\section{RESULTADOS}

- El costo del daño sobre las actividades económicas significativas del ACP Milpuj - La Heredad (apicultura y producción de tara), por el incendio provocado, equivale a $\mathbf{S} / \mathbf{4 5 , 9 2 7 . 4 1 .}$

- El costo de restauración, por los gastos incurridos para la reforestación del área afectada por el incendio provocado del ACP Milpuj - La Heredad, equivale a $\mathrm{S} / . \mathbf{1 3}, \mathbf{6 8 2 . 7 5}$.

- El valor de no uso, por los valores económicos de los servicios ecosistémicos que nos brinda los ecosistemas de Bosque Seco Premontano Tropical (bs-PT) y el Bosque Seco Montano Bajo Tropical (bs-MBT); equivale a $\mathrm{S} / . \mathbf{1 5 0 , 6 6 9 . 5 0 4}$.

- Finalmente, calculado el costo de los daños y costos de restauración, además, encontrado el valor de no uso de este tipo de ecosistema a través del mapa de los servicios ecosistémicos de Contanza et al. (1997), se encontró que el valor económico del daño total ocasionado sobre los 23.46 ha, por el incendio intencionado en el ACP Milpuj - La Heredad, equivale a $S / .210,279.67$; con un valor económico de daño de $\mathbf{S} / \mathbf{8 , 9 6 3 . 3 3 / h a . ~}$

\section{CONCLUSIONES}

1. El valor económico del daño a causa del incendio provocado en el ACP Milpuj - La Heredad, llega a ser una estimación por lo que los resultados deben tomarse como aproximaciones al valor real.

2. La cuantificación del daño tiene como fin de promover la restauración del bosque seco impactado y conservación del área no impactada, dentro del escenario actual de degradación y destrucción por el incendio intencionado.

3. Fin último de esta investigación a través de la valoración económica es la cuantificación, en términos monetarios, para la indemnización necesaria por el daño y perjuicios ocasionados sobre los valores de uso y no uso del ACP MilpujLa Heredad; o la implementación de un programa de restauración del ecosistema bosque seco, después del daño ambiental.

\section{DISCUSIÓN}

Los servicios ecosistémicos son aquellos procesos y funciones de los ecosistemas que son percibidos por las personas como un beneficio de tipo ecológico, cultural o económico, directo o indirecto.

Sin embargo, las externalidades que afectan los bienes o servicios ecosistémicos son comunes, sobre todo por el hecho que los daños ocasionados no tienen un costo para quien lo produce; y tampoco los individuos que se ven perjudicados reciben contraprestación alguna por el perjuicio causado (MINAMBIENTE, 2003).

Para ello, existen diversos procedimientos metodológicos para estimar, en términos monetarios, la pérdida de bienestar en las personas a causa de los impactos ambientales negativos (MINAM, 2013)

Siendo uno de ellos, la valoración económica, a través de métodos y técnicas basadas en la teoría económica, incorpora el análisis costo-beneficio de los impactos ambientales que afectan los bienes y servicios del capital natural; estimando los costos de su pérdida y beneficios sociales en unidades monetarias.

Pero tal cual señala Constanza et al., (1998), la valoración económica no deberías ser la única herramienta de decisión, sino una más entre una amplia gama de criterios para la toma de decisiones para los responsables de las decisiones.

Para el caso de esta investigación, el procedimiento metodológico para estimar, en términos monetarios, la pérdida de bienestar en las personas a causa de los impactos ambientales negativos se siguió el paso recomendado por el MINAM (2013) en la "Guía de Valoración Económica de Impactos Ambientales", el cual consiste en seleccionar los impactos ambientales negativos significativos, identificar la relación entre los impactos ambientales negativos significativos, identificar la relación entre los impactos y los agentes impactados y finalmente elegir el método de valoración y estimar los valores.

Los métodos de valoración económica utilizados en esta investigación, para encontrar el valor económico total del daño, tradujo el impacto ocasionado por el incendio en los valores de uso y no uso del capital natural en el ACP Milpuj La Heredad, llegando al fin último del análisis Costo - Beneficio que es la cuantificación de la indemnización necesaria por un daño ambiental percibido o el programa de restauración preferido después de un daño ambiental 


\section{REFERENCIAS BIBLIOGRÁFICAS}

CONGRESO DE LA REPÚBLICA. (1997). Ley Nº 26834. Ley de Áreas Naturales Protegidas. 17 de junio.

Constanza, R.; D'Arge, R.; De Groot, R.; Farber, S.; Grasso, M.; Hannon, B.; Limburg, K.; Naeem, S.; O'neill, R.; Paruelo, J.; Raskin, R.; Sutton, P. \& Belt, M. (1998). "The value of ecosystem services: putting the issues in perspective". Ecological Economics. Sweden, número 25, pp. 67-72.

Labandeira, X.; León, C. \& Vásquez, M. (2007). Economía ambiental. Madrid: Pearson Educación S.A.

Corcuera, C. (2018). Mapa del Área quemada del ACP-Milpuj-La Heredad. Chachapoyas.

Epiquién, M. (2009). Expediente técnico para el reconocimiento del área de conservación privada Milpuj-La Heredad. Chachapoyas.

GOBIERNO REGIONAL AMAZONAS, AUTORIDAD REGIONAL AMBIENTAL \& NATURALEZA \& CULTURA INTERNACIONAL. (2013). Expediente técnico para la creación de un área de conservación regional en el ámbito de los Bosques Tropicales Estacionalmente Secos del Marañón. Amazonas.

Labandeira, X.; León, C. \& Vásquez, M. (2007). Economía ambiental. Madrid: Pearson Educación S.A.

MINISTERIO DEL AMBIENTE (MINAM). (2013). Proyecto Guía de Valoración Económica de Impactos Ambientales. Lima: MINAM.

MINISTERIO DEL AMBIENTE (MINAM). (2014). Proyecto Guía de Valoración Económica del Patrimonio Natural. Lima: Dirección General de Evaluación, Valoración y Financiamiento del Patrimonio Natural.

MINISTERIO DEL AMBIENTE (MINAM). (2014). Ministerio del Ambiente reconoció 14 nueva Áreas de Conservación Privadas (ACP). Consulta: 22 de marzo de 2018. < http://www.minam.gob.pe/notas-deprensa/ministerio-del-ambiente-reconocio14-nuevas-areas-de-conservacion-privadasacp/>

MINISTERIO DE AMBIENTE, VIVIENDA Y DES A R R O L O TERRIT OR I A L (MINAMBIENTE). (2003). Guía Metodológica para la Valoración Económica de Bienes, Servicios Ambientales y Recursos Naturales. Colombia

NATURALEZA \& CULTURA INTERNACIONAL (NCI). (2017). "Proceso de Gestión para el reconocimiento del Área de Conservación
Regional (ACR) Bosque Tropical Estacionalmente seco del Marañón (BTESM)". Ponencia presentada en el Auditorio de la Municipalidad Provincial de Chachapoyas.

Pérez, O. (2008). Valoración Económica de los recursos y del medio ambiente. Lima.

POLICÍA NACIONAL DEL PERÚ - REGPOL Amazonas. (2017). Documento de Denuncia Caso 166-2017. Fuero Común / ambiental (delito) contra los recursos naturales / alteración del ambiente o paisaje. Tingo.

PUCP. (2013). "El daño ambiental en la Ley General del Ambiente”. Derecho PUCP. Lima, N 70, pp. 187-196.

SERVICIO NACIONAL DE ÁREAS NATURALES PROTEGIDAS POR EL ESTADO (SERNANP). (2018). Áreas de Conservación Privada. Consulta: 22 de marzo de 2018. $<$ http://www.sernanp.gob.pe/areas-deconservaciòn-privada $>$

SERVICIO NACIONAL DE ÁREAS NATURALES PROTEGIDAS POR EL ESTADO (SERNANP). (2011). ACP Milpuj - La Heredad: Reconocen el Áreas Conservación Privada Milpuj - La Heredad. Consulta: 22 de $\begin{array}{llllll}\mathrm{marz} & \mathrm{de} & 2 & 0 & 1 & 8 .\end{array}$ $<$ http://legislacionanp.org.pe/acp-milpuj-laheredad/>

SOCIEDAD PERUANA DE DERECHO AMBIENTAL (SPDA). (2012). MINAM reconoció trece áreas de conservación privada. Consulta: 22 de marzo de 2018 $<$ http://www.actualidadambiental.pe/?p=147 $17>$ 


\section{ANEXOS}

Anexo 1. Cálculo de Costos del daño sobre la producción de apicultura

\begin{tabular}{|c|c|c|c|c|c|c|}
\hline $\begin{array}{c}\text { ITE } \\
\text { M }\end{array}$ & DESCRIPCIÓN & UND & $\begin{array}{c}\text { Cantida } \\
\text { d }\end{array}$ & $\mathrm{N}^{0}$ de veces & $\mathbf{P U}$ & PARCIAL \\
\hline 1 & VENTA MIEL ABEJA - 2017 & & & & & S/ 20,400.00 \\
\hline 1.1 & Miel de abeja & $\begin{array}{c}\mathrm{kg} / \mathrm{añ} \\
\mathrm{O}\end{array}$ & 672 & 1 & $\mathrm{~S} / 25.00$ & S/ $16,800.00$ \\
\hline 1.2 & Mano de obra para cosecha de miel de abeja & $\mathrm{DH}$ & 45 & 2 & $\mathrm{~S} / 40.00$ & $\mathrm{~S} / 3,600.00$ \\
\hline 2 & MATERIALES PARA LA MIEL & & & & & $\mathrm{S} / \mathbf{3 4 0 . 0 0}$ \\
\hline 2.1 & Baldes de 20 litros & Und & 24 & 1 & $\mathrm{~S} / 10.00$ & $\mathrm{~S} / 240.00$ \\
\hline 2.2 & Potes o envases plásticos de $32 \mathrm{oz}$ ( 1 litro) & $\begin{array}{c}\text { Cient } \\
0 \\
\end{array}$ & 1 & 1 & $\mathrm{~S} / 100.00$ & $\mathrm{~S} / 100.00$ \\
\hline 3 & Mantenimiento de Colmenas & & & & & $\mathrm{S} / \mathbf{8 0 . 0 0}$ \\
\hline 3.1 & Mantenimiento de Colmenas & DH & 2 & 1 & $\mathrm{~S} / 40.00$ & $\mathrm{~S} / 80.00$ \\
\hline 4 & $\begin{array}{l}\text { INSUMOS, EQUIPOS Y MATERIALES } \\
\text { PARA LA OBTENCIÓN DE LA MIEL DE } \\
\text { ABEJA }\end{array}$ & & & & & $\mathrm{S} / \mathbf{1 , 1 2 5 . 0 0}$ \\
\hline 4.1 & $\begin{array}{l}\text { Vestimenta de apicultor (velo con sombrero, } \\
\text { overol blanco, guantes y zapatos de protección) }\end{array}$ & Jgo & 1 & 1 & $\mathrm{~S} / 100.00$ & $\mathrm{~S} / 100.00$ \\
\hline 4.2 & Palanca & Und & 1 & 1 & $\mathrm{~S} / 25.00$ & $\mathrm{~S} / 25.00$ \\
\hline 4.3 & Escobilla & Und & 1 & 1 & $\mathrm{~S} / 10.00$ & $\mathrm{~S} / 10.00$ \\
\hline 4.4 & Desoperculador & Und & 1 & 1 & S/ 10.00 & S/ 10.00 \\
\hline 4.5 & Ahumador & Und & 1 & 1 & $\mathrm{~S} / 40.00$ & $\mathrm{~S} / 40.00$ \\
\hline 4.6 & Cera & $\mathrm{kg}$ & 3 & 1 & S/ 80.00 & $\mathrm{~S} / 240.00$ \\
\hline 4.7 & Centrífuga & Gbl & 1 & 1 & $\mathrm{~S} / 400.00$ & $\mathrm{~S} / 400.00$ \\
\hline 4.8 & Cerificador solar & Gbl & 1 & 1 & $\mathrm{~S} / 300.00$ & S/ 300.00 \\
\hline 5 & COSTO DE PÉRDIDA DE APIARIO & & & & & $\mathrm{S} / \mathbf{3 , 4 2 5 . 0 0}$ \\
\hline 5.1 & Abejas & $\begin{array}{c}\text { Núcle } \\
\text { o }\end{array}$ & 1 & 4 & $\mathrm{~S} / 250.00$ & S/ $1,000.00$ \\
\hline 5.2 & Colmena & $\mathrm{Gbl}$ & 1 & 4 & S/ 200.00 & S/ 800.00 \\
\hline 5.3 & Miel & $\mathrm{kg}$ & 16.25 & 4 & $\mathrm{~S} / 25.00$ & S/ $1,625.00$ \\
\hline & TOTA & & & & & S/ $25,370.00$ \\
\hline
\end{tabular}

Anexo 2. Cálculo de Costos del daño sobre la producción de tara

\begin{tabular}{|c|c|c|c|c|c|c|}
\hline ITEM & DESCRIPCIÓN & UND & $\begin{array}{c}\text { Metrad } \\
\text { o }\end{array}$ & $\begin{array}{l}N^{0} \text { de } \\
\text { veces }\end{array}$ & PU & PARCIAL \\
\hline 1 & PRODUCTIVIDAD DE TARA & & & & & S/ 6,666.67 \\
\hline 1.1 & Cosecha de tara & $\mathrm{Tn}$ & 2.00 & 1 & $\mathrm{~S} / 2,500.00$ & S/ 5,000.00 \\
\hline 1.2 & Mantenimiento (poda, raleo) & $\mathrm{Gbl}$ & 1.00 & 1 & S/ 1,666.67 & $\mathrm{S} / 1,666.67$ \\
\hline 2 & PÉRDIDA POR EL IMPACTO & & & & & S/ 1,666.67 \\
\hline 2.1 & Costo del área afectada & $\mathrm{Gbl}$ & 1.00 & 1 & S/ $1,666.67$ & S/ 1,666.67 \\
\hline 3 & $\begin{array}{l}\text { RESERVORIO PARA RIEGO CON } \\
\text { GEOMEMBRANA }\end{array}$ & & & & & S/ 12,224.07 \\
\hline 3.1 & ESTRUCTURAS & & & & & S/ 12,224.07 \\
\hline 3.1.1 & OBRAS PRELIMINARES & & & & & S/ 683.65 \\
\hline 3.1.1.1 & Limpieza manual del terreno & $\mathrm{m} 2$ & 121.00 & & $\mathrm{~S} / 1.03$ & S/ 124.63 \\
\hline 3.1.1.2 & Trazo y replanteo & $\mathrm{m} 2$ & 121.00 & & $\mathrm{~S} / 4.62$ & S/ 559.02 \\
\hline 3.1 .2 & MOVIMIENTO DE TIERRAS & & & & & S/ 7,826.04 \\
\hline 3.1.2.1 & Excavación para reservorio & $\mathrm{m} 3$ & 266.20 & & $\mathrm{~S} / 15.94$ & $\mathrm{~S} / 4,243.23$ \\
\hline 3.1.2.2 & Eliminación del material excedente & $\mathrm{m} 3$ & 266.20 & & $\mathrm{~S} / 12.75$ & $\mathrm{~S} / 3,394.05$ \\
\hline 3.1.2.3 & Nivelación interior y apisonado & $\mathrm{m} 2$ & 121.00 & & $\mathrm{~S} / 1.56$ & $\mathrm{~S} / 188.76$ \\
\hline 3.1 .3 & RESERVORIO CON GEOMEMBRANA & & & & & S/ 1,392.38 \\
\hline 3.1.3.1 & Colocación de geomembrana & $\mathrm{m} 2$ & 217.56 & & S/ 6.40 & $\mathrm{~S} / 1,392.38$ \\
\hline 3.1.4 & RED DE DISTRIBUCIÓN & & & & & S/ 2,247.00 \\
\hline 3.1.4.1 & Tubería PVC 4" & $\mathrm{m}$ & 50.00 & & $\mathrm{~S} / 44.94$ & $\mathrm{~S} / 2,247.00$ \\
\hline 3.1.5 & FLETE TERRESTRE & & & & & S/ 75.00 \\
\hline 3.1.5.1 & Flete terrestre de geomembrana & Gbl & 1.00 & & $\mathrm{~S} / 55.00$ & $\mathrm{~S} / 55.00$ \\
\hline 3.1.5.2 & Flete terrestre de tubería y accesorios de PVC & Gbl & 1.00 & & $\mathrm{~S} / 20.00$ & $\mathrm{~S} / 20.00$ \\
\hline
\end{tabular}


Anexo 3. Cálculo de Costo de restauración total por reforestación

\begin{tabular}{|c|l|c|c|c|c|c|}
\hline $\begin{array}{c}\text { ITE } \\
\mathbf{M}\end{array}$ & \multicolumn{1}{|c|}{ DESCRIPCIÓN } & $\begin{array}{c}\text { UN } \\
\mathbf{D}\end{array}$ & $\begin{array}{c}\text { Cantida } \\
\mathbf{d}\end{array}$ & $\begin{array}{c}\mathbf{N}^{\mathbf{0}} \mathbf{d e} \\
\mathbf{v e c e s}\end{array}$ & PU & PARCIAL \\
\hline $\mathbf{1}$ & REFORESTACIÓN & & & & & \\
\hline $\mathbf{1 . 1}$ & $\begin{array}{l}\text { Plantones de cedro kuelapense, tara, aliso, } \\
\text { balsa, mutuy, guayo, pavicuna, huarango y } \\
\text { eucalipto }\end{array}$ & Und & 1600 & 1 & $\mathrm{~S} / 1.00$ & $\mathrm{~S} / 1,600.00$ \\
\hline $\mathbf{2}$ & FLETE & & & & & \\
\hline $\mathbf{2 . 1}$ & Transporte terrestre de plantones forestales & $\mathrm{Gbl}$ & 1 & 1 & $\mathrm{~S} / 142.22$ & $\mathrm{~S} / 142.22$ \\
\hline $\mathbf{3}$ & JORNAL & & & & & \\
\hline $\mathbf{3 . 1}$ & Labores de reforestación & $\mathrm{DH}$ & 26 & 4 & $\mathrm{~S} / 40.00$ & $\mathrm{~S} / 4,160.00$ \\
\hline $\mathbf{4}$ & LABORES DE DESHIERBA & & & & & \\
\hline $\mathbf{4 . 1}$ & Deshierba & $\mathrm{DH}$ & 12 & 3 & $\mathrm{~S} / 40.00$ & $\mathrm{~S} / 1,440.00$ \\
\hline $\mathbf{5}$ & ABONAMIENTO & & & & & \\
\hline $\mathbf{5 . 1}$ & Abono & $\begin{array}{c}\text { Cost } \\
\mathrm{al}\end{array}$ & 10 & 1 & $\mathrm{~S} / 50.00$ & $\mathrm{~S} / 500.00$ \\
\hline $\mathbf{6}$ & RIEGO EN TEMPORADA SECA & & & & & \\
\hline $\mathbf{6 . 1}$ & Requerimiento de agua & $\mathrm{m} 3$ & 105.14 & 1 & $\mathrm{~S} / 55.55$ & $\mathrm{~S} / 5,840.53$ \\
\hline
\end{tabular}

\section{Anexo 4. Panel fotográfico}

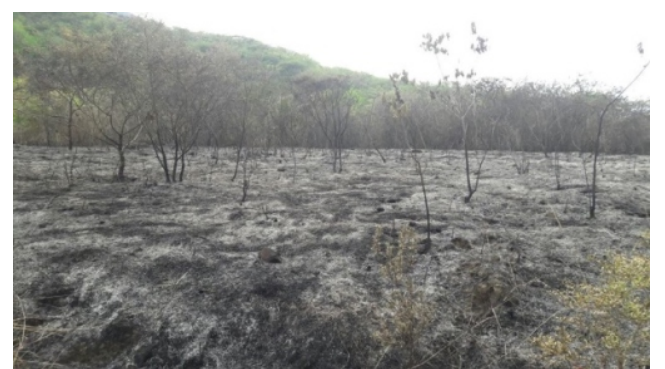

Fuente: Heredia,2017

Fotografía 01: Plantones de tara afectados por el incendio.

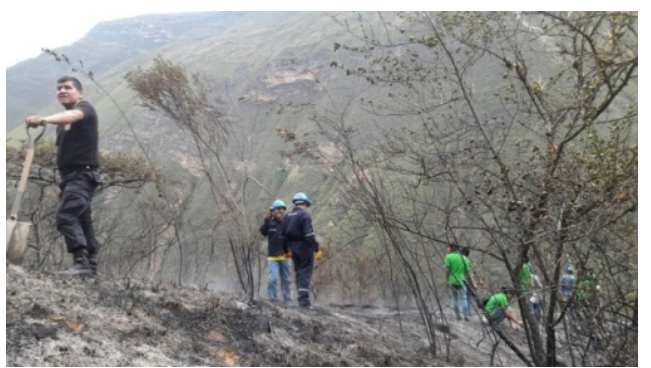

Fuente: Heredia,2017

Fotografía 03: Intentos de extinguir el fuego.

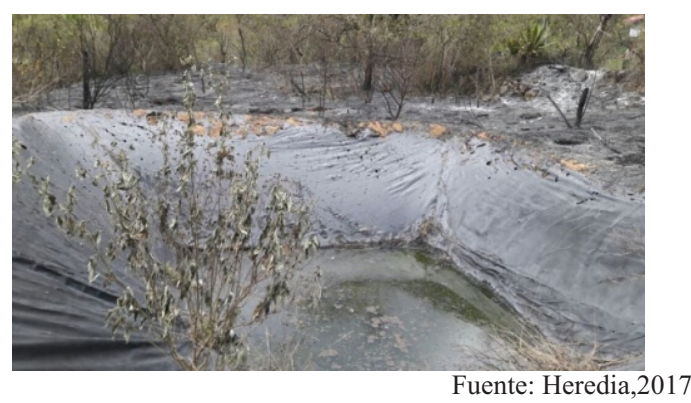

Fotografía 02: Daño del reservorio por el incendio.

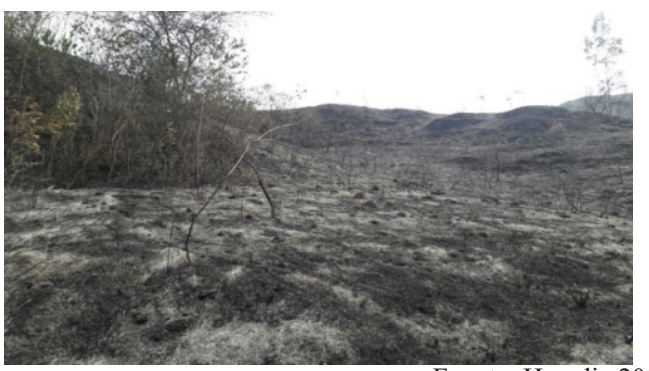

Fuente: Heredia,2017

Fotografía 04: Daño de la zona sur por el incendio ocasionado.

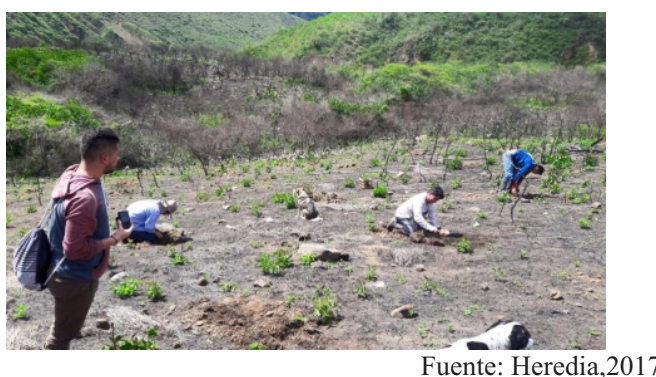

Fotografía 05: Actividades de restauración del ecosistema dañado por el incendio ocasionado. 